\title{
Erratum to: Impact of intra-abdominal absorbable sutures on surgical site infection in gastrointestinal and hepato-biliary-pancreatic surgery: results of a multicenter, randomized, prospective, phase II clinical trial
}

\author{
Yoshihiko Maehara $^{1} \cdot$ Ken Shirabe $^{1} \cdot$ Shunji Kohnoe $^{1} \cdot$ Yasunori Emi $^{1} \cdot$ Eiji Oki $^{1} \cdot$ Yoshihiro Kakeji $^{2} \cdot$ Hideo Baba $^{3} \cdot$ \\ Masataka Ikeda $^{4} \cdot$ Michiya Kobayashi $^{5} \cdot$ Tadatoshi Takayama $^{6} \cdot$ Shoji Natsugoe $^{7} \cdot$ Masashi Haraguchi $^{8}$. \\ Kazuhiro Yoshida ${ }^{9} \cdot$ Masanori Terashima $^{10} \cdot$ Mitsuru Sasako $^{11} \cdot$ Hiroki Yamaue $^{12}$ - Norihiro Kokudo $^{13}$. \\ Katsuhiko Uesaka $^{14}$ - Shinji Uemoto ${ }^{15}$ Tomoo Kosuge ${ }^{16}$ - Yoshiki Sawa ${ }^{17}$ - Mitsuo Shimada ${ }^{18}$ - Yuichiro Doki ${ }^{19}$. \\ Masakazu Yamamoto ${ }^{20}$. Akinobu Taketomi ${ }^{21}$ - Masahiro Takeuchi ${ }^{22} \cdot$ Kouhei Akazawa $^{23}$. Takeharu Yamanaka ${ }^{24}$. \\ Mototsugu Shimokawa ${ }^{25}$
}

Published online: 27 September 2017

(C) Springer Japan KK 2017

\section{Erratum to: Surg Today (2017) 47:1060-1071 DOI 10.1007/s00595-017-1480-3}

In the original publication, the article category was published as "Review Article". The correct category should read as "Original Article".

The online version of the original article can be found under doi:10.1007/s00595-017-1480-3.

\section{Ken Shirabe}

kshirabe@surg2.med.kyushu-u.ac.jp

1 Department of Surgery and Science, Kyushu University, 3-1-1 Maidashi, Higashi-ku, Fukuoka 812-8582, Japan

2 Division of Gastrointestinal Surgery, Department of Surgery, Kobe University, Kobe, Japan

3 Department of Gastroenterological Surgery, Graduate School of Medical Sciences, Kumamoto University, Kumamoto, Japan

4 Department of Gastroenterological Surgery, Graduate School of Medicine, Osaka University, Osaka, Japan

5 Department of Human Health and Medical Sciences, Hospital Administration Section, Kochi Medical School, Kochi, Japan

6 Department of Digestive Surgery, Nihon University School of Medicine, Tokyo, Japan

7 Department of Digestive Surgery, Breast and Thyroid Surgery, Graduate School of Medicine, Kagoshima University, Kagoshima, Japan

8 Department of Surgery, Nagasaki University Graduate School of Biomedical Science, Nagasaki, Japan
9 Department of Surgical Oncology, Graduate School of Medicine, Gifu University, Gifu, Japan

10 Division of Gastric Surgery, Shizuoka Cancer Center, Shizuoka, Japan

11 Department of Surgery, Hyogo College of Medicine, Nishinomiya, Japan

12 Second Department of Surgery, School of Medicine, Wakayama Medical University, Wakayama, Japan

13 Hepato-Biliary-Pancreatic Surgery Division, Artificial Organ and Transplantation Division, Department of Surgery, Graduate School of Medicine, University of Tokyo, Tokyo, Japan

14 Division of Hepato-Biliary-Pancreatic Surgery, Shizuoka Cancer Center, Shizuoka, Japan

15 Division of Hepato-Biliary-Pancreatic Surgery and Transplantation Department of Surgery, Graduate School of Medicine, Kyoto University, Kyoto, Japan

16 Hepatobiliary and Pancreatic Surgery Division, National Cancer Center Hospital, Tokyo, Japan

17 Department of Cardiovascular Surgery, Graduate School of Medicine, Osaka University, Oksaka, Japan 
18 Department of Surgery, The University of Tokushima, Tokushima, Japan

19 Department of Gastroenterological Surgery, Graduate School of Medicine, Osaka University, Osaka, Japan

20 Department of Surgery, Institute of Gastroenterology, Tokyo Women's Medical University, Tokyo, Japan

21 Department of Gastroenterological Surgery I, Graduate School of Medicine, Hokkaido University, Sapporo, Japan

22 Department of Clinical Medicine (Biostatistics and Pharmaceutical Medicine), School of Pharmacy, Kitasato University, Tokyo, Japan

23 Department of Medical Informatics, Niigata University Medical and Dental Hospital, Niigata, Japan

24 Department of Biostatistics, Yokohama City University, Yokohama, Japan

25 Department of Cancer Information Research, Kyushu Cancer Center, Fukuoka, Japan 